\title{
Pathologische Frakturen der Brust- und Lendenwirbelsäule
}

\author{
Erich Hartwig, Michael Schulte, Markus Schultheiss, Michael Kramer, Lothar Kinzl
}

\section{Zusammenfassung}

Pathologische Wirbelfrakturen sind überwiegend Folge von Wirbelmetastasen. Prädilektionsorte der Metastasierung sind Brust- und Lendenwirbelsäule mit Schwerpunkt im thorakolumbalen Übergang. Die Prognose der Patienten mit ossär metastasierten Tumoren hat sich durch die interdisziplinäre Diagnostik und multimodale Therapie der Tumorerkrankung erheblich verbessert. Ziel einer chirurgischen Therapie muss es sein, das operative Verfahren strategisch angepasst an Lebenserwartung, Tumorbiologie und den weiteren therapeutischen Möglichkeiten auszuwählen, um die Lebensqualität des Patienten zu verbessern. Absolute Operationsindikationen stellen neurologische Ausfalls- erscheinungen dar, nur die frühzeitige operative Revision kann Dauerschäden minimieren. Instabilitäten des Achsenskelettes implizieren Schmerzen, Deformitäten und drohende neurologische Defizite. Sie erfordern in der Regel ebenfalls die operative Korrektur und Stabilisierung der Deformität. Operative Maßnahmen bei tumorösen Veränderungen an der Wirbelsäule reichen von der reinen Stabilisierung bis zur Vertebrektomie mit ventrodorsaler Stabilisation. Die Radikalität des Eingriffes ist abhängig vom Zustand des Patienten, der Prognose und weiteren therapeutischen Möglichkeiten. Oberstes Ziel der operativen Maßnahme muss eine Verbesserung der Lebensqualität des Patienten sein.

\section{Einleitung:}

Unter pathologischen Frakturen versteht man Knochenbrüche, die in einem erkrankten und deshalb geschwächten Skelettabschnitt ohne adäquates Trauma auftreten. Hauptursachen für pathologische Frakturen der Wirbelsäule sind einerseits die senile Osteoporose und primäre Knochentumoren, zum großen Teil jedoch sekundäre d.h. metastatische Knochentumoren.

Osteoporotische Frakturen bedürfen in der Regel keiner lokalen Therapie. Bei bestehender Deck- und Bodenplattenimpression sind diese in der Regel stabile Frakturformen.

OP-JOURNAL 2001; 17: 191-195

(c) Georg Thieme Verlag Stuttgart · New York
Operative Stabilisierungsmaßnahmen stellen in diesen Fällen eine Ausnahmeindikation dar und bedürfen aufgrund der Knochenqualität einer eingehenden Planung, insbesondere hinsichtlich der notwendigen Verankerung von Implantatmaterial.

Tumorbedingte pathologische Frakturen führen zu einer Wirbelsäulendeformität und Instabilität. Nervenwurzel- bzw. Rückenmarkkompressionen können zu entsprechenden klinisch-neurologischen Ausfällen bis hin zum Querschnittssyndrom führen. Von dieser primär neurologischen Schädigung muss eine sekundäre Beeinträchtigung der neuralen Strukturen abgegrenzt werden, die durch chronische Rückenmarküberdehnung in der Folge einer meist kyphotischen Knickbildung auftreten kann. Die Durchblutung des Rückenmarkes kann aufgrund eines „Steal-Effektes“ des Tumors reduziert sein und so zu einer Vorschädigung des Rückenmarkes führen.
Die Prognose von Patienten mit ossär metastasierenden Tumoren hat sich in den letzten 30 Jahren erheblich verbessert. Ursächlich für diese Entwicklung ist vor allem die interdisziplinäre Diagnostik und multimodale Therapie dieser Erkrankung mit gezielter Chemo-, Immun-, Hormon-, Strahlentherapie und Chirurgie. Hierbei stellt die Wirbelsäule mit Brust- und Lendenwirbelsäule den häufigsten Manifestationsort der Metastasierung dar. In einer Sektionsstudie von Wong (1999) fanden sich bei 36\% von 832 Patienten Metastasen der Wirbelsäule.

Die chirurgischen Maßnahmen im Bereich der Wirbelsäule sind in der Regel als palliative Eingriffe zu werten. Weite Resektionen sind aufgrund der Limitierung durch das Rückenmark in der Regel nicht möglich. Ziel der chirurgischen Therapie muss daher ein für den Patienten möglichst wenig belastender Eingriff sein. Er sollte die Notwendigkeit einer Schmerztherapie reduzieren, neurologische Komplikationen verhindern, die Stabilität des Achsenorganes erhalten und die Lebensqualität des Patienten durch Erhalt der Mobilität gewährleisten.

So bedarf die chirurgische Therapie einer eingehenden Planung. Diese kann von einer reinen Entlastung des Rückenmarkes bis hin zu einer Tumorresektion im Sinne der Korporektomie oder kompletten Vertebrektomie mit Ersatz des resezierten Wirbelkörpers reichen. Bei bestehender Wirbelmetastasierung und fehlender Instabilität bzw. neurologischen Ausfallserscheinungen müssen die Möglichkeiten einer multimodalen Therapie mit gezielter Chemotherapie bzw. einer lokalen Strahlentherapie diskutiert werden. 
Pathologische Wirbelfrakturen sind häufig Folge einer metastasierenden Tumorerkrankung. Neurologische Ausfallserscheinungen können Folge einer direkten Tumorinvasion, einer Deformität oder lokaler Durchblutungsstörungen sein. Die Planung operativer Maßnahmen muss die Möglichkeiten multimodaler Therapiekonzepte berücksichtigen.

\section{Epidemiologie}

Tumorbedingte pathologische Wirbelfrakturen sind überwiegend Folge der Skelettmetastasierung eines Karzinoms, eines Plasmozytoms oder eines malignen Lymphoms. In der Gruppe der benignen Wirbeltumoren bzw. tumorähnlichen Veränderungen ist beim eosinophilen Granulom bzw. bei der LangerhansZellhistiozytose mit einer Fraktur zu rechnen. Pathologische Wirbelfrakturen bei spinalen Sarkomen stellen eine Rarität dar.

Bei den sekundären Wirbeltumoren stehen die so genannten osteophilen Primärtumoren Mammakarzinom, Bronchialkarzinom, Prostatakarzinom, Schilddrüsenkarzinom sowie Plasmozytome und Lymphome im Vordergrund. Zusammen mit den Karzinomen mit unbekanntem Primärtumor (carcinoma of unknown primary) machen diese Neubildungen im eigenen Krankengut 74\% der Patienten mit pathologischen Wirbelfrakturen aus. Prädilektionsort der ossären Metastasierung ist die Brustwirbelsäule, dabei ist die untere Brustwirbelsäule häufiger als die obere betroffen. Die Metastasierungshäufigkeit für die Wirbelsäulenabschnitte BWS und LWS ist mit $3: 2$ anzugeben mit der Dominanz des thorakolumbalen Überganges.

Das Risiko einer pathologischen Fraktur korreliert mit der Morphologie des Tumors. Ein osteoplastischer Metastasierungstyp ist repräsentativ für das Prostatakarzinom. Ossäre Metastasen dieses Tumors besitzen ein niedrigeres Frakturrisiko als Metastasen gemischter Typen (Mammakarzinom). Eine rein osteolytische Metastasierungsform ist typisch für Hypernephrome, Plasmozytome und Lymphome.

Aufgrund der Genese pathologischer Frakturen liegt der Altersgipfel dieser Patienten im 6. Lebensjahrzehnt, die Überlebenszeiten von Patienten mit pathologischen Frakturen der Brust- und Lendenwirbelsäule liegen im Median zwischen 3 Monaten beim Bronchialkarzinom und 3
Jahren beim Schilddrüsenkarzinom. Bei einzelnen Patienten kann die Überlebenszeit bis zu 10 Jahren oder länger betragen.

Leitsymptom einer pathologischen Fraktur der Brust- und Lendenwirbelsäule ist der Rückenschmerz. Resultierend aus der Häufigkeit bestehender Rückenproblematiken kommt es häufig zu einer verzögerten Diagnosestellung. So führen tumorbedingte Schmerzsymptomatiken bei benignen Tumoren erst nach durchschnittlich 18 Monaten, bei Sarkomen und Wirbelmetastasen durchschnittlich nach 10 Monaten zur Diagnosestellung einer Tumorerkrankung. 20-50\% der Patienten werden bereits mit neurologischen Ausfallerscheinungen zur chirurgischen Therapie vorgestellt. So ist die Forderung zu erheben, dass bei einem Patienten jenseits des 40.Lebensjahres mit einem Malignom in der Vorgeschichte bis zum Beweis des Gegenteiles der Verdacht einer Metastase der Wirbelsäule gestellt werden muss. Die Zeitspanne zwischen Schmerzbeginn und ersten neurologischen Symptomen stellt ein prognostisch bedeutsames Maß für die Wachstumsgeschwindigkeit des Tumors dar. Sie schwankt zwischen 4 Wochen beim Bronichalkarzinom und 6 bis 12 Monaten beim Mammakarzinom mit Low-risk-Metastasierung bzw. beim differenzierten Schilddrüsenkarzinom. Die neurologische Symptomatik ist in der Regel Zeichen einer Kompression durch direktes Tumorwachstum oder bedingt durch kyphotische Knickbildung auf dem Boden der pathologischen Fraktur und einer konsekutiven Dehnung des Rückenmarkes.

Nahezu jeder zweite Karzinompatient zeigt im Stadium der Metastasierung Knochenmetastasen. Wirbelmetastasen finden sich in $60 \%$ bei Patienten mit ossärer Disseminierung. Prädilektionsort der Wirbelsäule ist der thorakolumbale Übergang.

\section{Diagnostik}

Die Diagnose einer pathologischen Fraktur lässt sich meist aus der Übersichtsradiographie stellen. Die Computertomographie erlaubt durch Darstellung der knöchernen Strukturen eine Abschätzung einer bestehenden Instabilität. Die Kernspintomographie ermöglicht die dreidimensionale Darstellung der Tumorausdehnung, insbesondere der Ausbreitung im Spinalkanal und den paravertebralen Weichteilen. Durch Gadolinium-
Sequenzen lässt sich die Vaskularität des Tumors abschätzen. Mit der Möglichkeit der längerstreckigen Untersuchung kann ein diffuser Wirbelsäulenbefall dargestellt werden. Bei kernspintomographischem Nachweis einer stark vaskularisierten Metastase sollte bei Planung einer ausgedehnten Tumorresektion die Durchführung einer digitalen Subtraktions-Angiographie mit intraarterieller Tumorembolisation diskutiert werden. Diese erscheint bei Metastasen von hypernephroiden Karzinomen und Schilddrüsenkarzinomen sinnvoll.

Die Durchführung der Szintigraphie erlaubt als Screening-Methode den Nachweis weiterer Knochenmetastasen, ist jedoch für die Durchführung der chirurgischen Intervention an der Wirbelsäule nicht generell erforderlich. Da eine pathologische Fraktur mit Instabilität bzw. neurologischen Komplikationen eine dringliche Operationsindikation darstellt, muss die erforderliche Diagnostik notfallmäßig durchgeführt werden und insgesamt limitiert bleiben. Zusatzuntersuchungen wie Mehrphasen-Skelettszintigraphie, Knochenmark-Szintigraphie, Positronen-Emissions-Tomographie bringen hier in der Regel keine zusätzliche Entscheidungshilfe.

Die Biopsie als invasive diagnostische Maßnahme kann indiziert sein bei stabilen Frakturen und fehlenden neurologischen Ausfallserscheinungen bei Patienten, bei denen kein Primärtumor bekannt ist. Hier steht die computertomographische transpedikuläre Nadelpunktion bzw. operative transpedikuläre Biopsie zur Verfügung. Bedacht werden muss hierbei, dass durch den transpedikulären Zugang eine Kontamination der dorsalen Anteile erfolgt, welche bei einer späteren Tumorresektion kaum vollständig entfernt werden können.

In jedem Einzelfall muss entschieden werden, ob nur eine Stabilisierung mit Dekompression des Spinalkanales durchgeführt werden soll oder ob eine zusätzliche Korporektomie indiziert ist. Als Entscheidungshilfe wurde von Tokuhashi ein Scoring-System vorgeschlagen, welches Allgemeinzustand, Anzahl der knöchernen Läsionen außerhalb der Wirbelsäule, die knöchernen Läsionen in der Wirbelsäule, Organmetastasen, Sitz des Primärtumors und neurologische Störungen bewertet (siehe Tab.1).

Nicht berücksichtigt sind Plasmozytome und Lymphome, hier muss die Strahlen- 
Tab. 1 Evaluationsscore zur Prognoseabschätzung von Wirbelmetastasen (Tokuhashi). Die Resektion der Metastase wird ab einer Punktzahl von weniger als 9 vorgeschlagen

\begin{tabular}{lll}
\hline Allgemeinzustand & schlecht & 0 \\
& mäßig & 1 \\
knt & $\geq 3$ & 2 \\
knöcherne Läsionen & $1-2$ & 0 \\
außerhalb der Wirbelsäule & 0 & 1 \\
knöcherne Läsionen & $\geq 3$ & 2 \\
in der Wirbelsäule & $1-2$ & 0 \\
& 0 & 1 \\
Organmetastasen & nicht resektabel & 2 \\
& resektabel & 0 \\
Sitz des Primärtumors & keine Metastasen & 1 \\
& Lunge, Magen & 2 \\
& Niere, Leber, Uterus & 0 \\
& carcinoma of unknown primary & 1 \\
Schilddrüse, Prostata, Mamma, & 2 \\
Rektum & \\
neurologische Ausfallerscheinungen & Transversalsyndrom (Frankel A, B) \\
& Inkomplettes Transversalsyndrom & 0 \\
& (Frankel C, D) & 1 \\
& neurologisch unauff. & 2 \\
Maximum & & 12
\end{tabular}

therapie Eingang in das Behandlungskonzept finden.

Die Diagnose einer pathologischen Fraktur lässt sich meist aus der Übersichtsradiographie stellen. Die Computertomographie erlaubt durch Darstellung der knöchernen Strukturen eine Abschätzung einer bestehenden Instabilität. Die Kernspintomographie ermöglicht die dreidimensionale Darstellung der Tumorausdehnung, insbesondere der Ausbreitung im Spinalkanal und den paravertebralen Weichteilen. Bei kernspintomographischem Nachweis einer stark vaskularisierten Metastase sollte bei Planung einer ausgedehnten Tumorresektion die Durchführung einer digitalen Subtraktions-Angiographie mit intraarterieller Tumorembolisation diskutiert werden. Diese erscheint bei Metastasen von hypernephroiden Karzinomen und Schilddrüsenkarzinomen sinnvoll.

\section{Operationsindikationen}

Für die Operationsindikation der pathologischen Frakturen von Brust- und Lendenwirbelsäule gelten primär die Richtlinien der Traumatologie der Wirbelsäule. Als Notfallindikation gilt die Entwicklung eines Transversalsyndromes oder ConusCaudasyndromes. Da die Rückbildungstendenz motorischer und vegetativer Ausfallerscheinungen als schlecht einzuZeilung pathologischer Wirbelsäule nicht auszugehen ist, kann eine operative Maßnahme nur bei zu erwartender Strahlen- oder Chemosensibilität (Beisp.: Plasmozytom) umgangen werden.

Ziel der operativen Maßnahme ist der Erhalt der Lebensqualität und Mobilität des

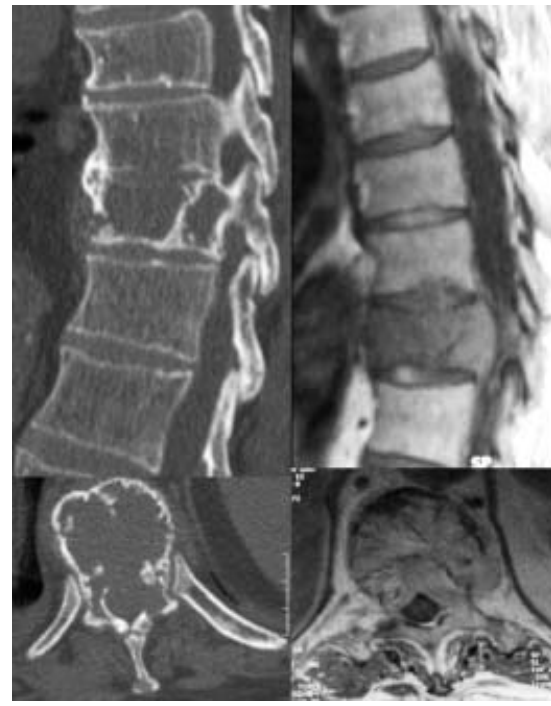

Abb. 1 a Computertomographisch wabenartige osteolytische Destruktion von BWK 10 bei einem 69-jährigen Patienten mit inkomplettem Transversalsyndrom. Radiomorphologisch typisch für Plasmozytom.
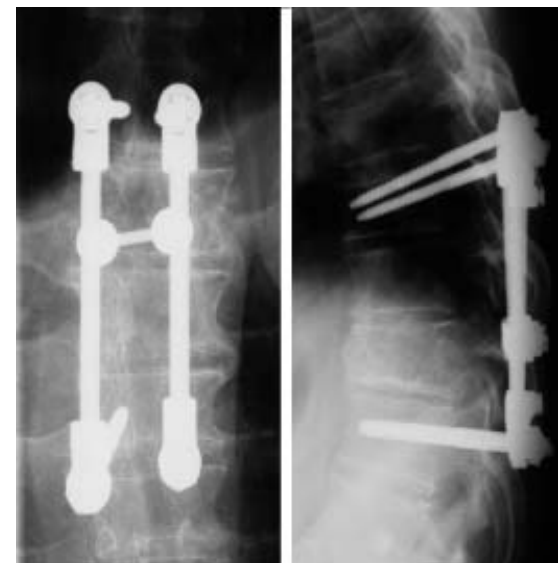

Abb.1b Notfallmäßig Durchführung einer Laminektomie mit dorsaler Spondylodese. Histologisch Bestätigung der Diagnose. Zusätzliche Strahlen- und hämatologische Therapie eingeleitet.

Patienten. Die Schmerzsymptomatik des Patienten ist daher in der Indikationsstellung zu berücksichtigen. Die operative Maßnahme kann den Schmerzmittelbedarf reduzieren, durch die wiedergewonnene Mobilität des Patienten können Sekundärkomplikationen wie Thrombosen und Pneumonien bedingt durch Immobilisation vermieden werden.

Eingang in die Operationsplanung finden Entität und Prognose der Grunderkrankung. In Zusammenarbeit mit Onkologen und Strahlentherapeuten muss das therapeutische Management der Patienten mit Wirbeltumoren auch hinsichtlich der Radikalität des Eingriffes festgelegt werden. 
Für die Operationsindikation der pathologischen Frakturen von Brust- und Lendenwirbelsäule gelten primär die Richtlinien der Traumatologie der Wirbelsäule. Als Notfallindikation gilt die Entwicklung eines Transversalsyndromes, da die Rückbildungstendenz motorischer Ausfallerscheinungen als schlecht einzustufen ist. Bei der Indikationsstellung zu operativen Maßnahmen müssen interdisziplinäre therapeutische Möglichkeiten Berücksichtigung finden.

\section{Operative Strategie und Zugangswege:}

Eine Tumorresektion im Bereich der Wirbelsäule mit dem Ziel eines weiten Sicherheitsabstandes ist aufgrund der anatomischen Beziehung zum Rückenmark nur in seltenen Fällen möglich, in den meisten Fällen handelt es sich um eine intraläsionale Resektion mit einer R1-Situation. Der kurative Therapieansatz besonders in der Metastasenchirurgie ist daher selten und besteht lediglich bei solitärer Metastase eines multimodal gut kontrollierbaren Primärtumors. Operationsziele sind in erster Linie die Dekompression der neuralen Strukturen, die Beseitigung der Wirbelsäulendeformität und die Stabilisierung des Achsenorganes.

Die Dekompression des Rückenmarkes und der Nervenwurzeln ist über den dorsalen Zugang mit Durchführung einer erweiterten interlaminären Fensterung bis hin zur Laminektomie möglich. Die Vorteile des dorsalen Zuganges liegen in der für den Patienten geringen Invasivität. Anwendung findet dieser in hochpalliativen Situationen, bei mehrsegmentalem Tumorbefall und bei multimodal zu kontrollierenden Tumoren (Abb.1).

Nachteile bestehen in der begrenzten Möglichkeit der Tumorresektion und der weiteren Destabilisierung der Wirbelsäule durch resezierende Maßnahmen (Laminektomie).

Es sollte daher immer eine zusätzliche transpedikuläre Instrumentation durch eines der zahlreich verfügbaren Implantate erfolgen. Zur Begrenzung des Funktionsverlustes wird diese so kurzstreckig wie möglich erfolgen. Die Verankerung des Implantates erfordert eine stabile Knochenqualität.

Da Metastasen in der Wirbelsäule in der Regel im Wirbelkörper lokalisiert sind, wäre der ventrale Zugang zu Metastasenresektion prinzipiell zu bevorzugen. An
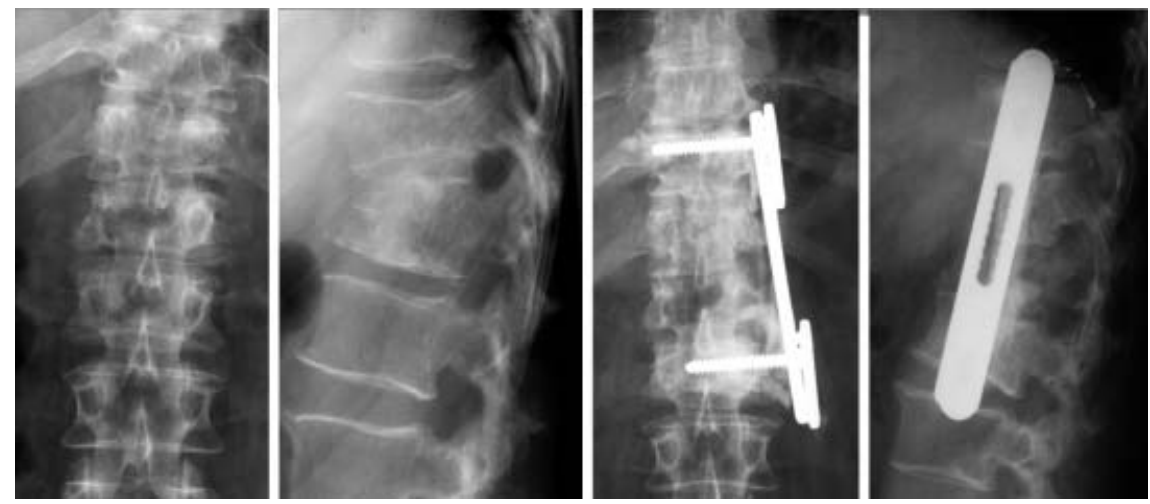

Abb. 2 Echinokokkusbefall der Wirbelsäule mit pathologischer Fraktur von LWK1. Operative Ausräumung, Spaninterposition und systemische Therapie.
Brust- und Lendenwirbelsäule mit Hauptlokalisation im thorakolumbalen Übergang bedeutet dieses in der Regel die Durchführung eines transthorakalen Zuganges. Auch unter Berücksichtigung minimalinvasiver Verfahren muss als wesentlicher Nachteil die höhere Belastung des Patienten durch pulmonale Komplikationen gesehen werden.

Der ventrale Zugang wird bevorzugt bei prognostisch günstigen pathologischen Wirbelfrakturen (Abb. 2) wie dem metas- tasierten Mamma- und Schilddrüsenkarzinom. Operationstechnisch sollten nicht mehr als 2 benachbarte Wirbelkörper betroffen sein. Der Aufbau der ventralen Säule erfogt durch Wirbelkörperersatzsysteme mit einer zusätzlichen Instrumentation. Die Verwendung kortikospongiöser Beckenkammspäne muss in der Tumorchirurgie wegen der Gefahr von Lokalrezidiven und notwendiger Strahlentherapie als fragwürdig erscheinen (Abb.2).
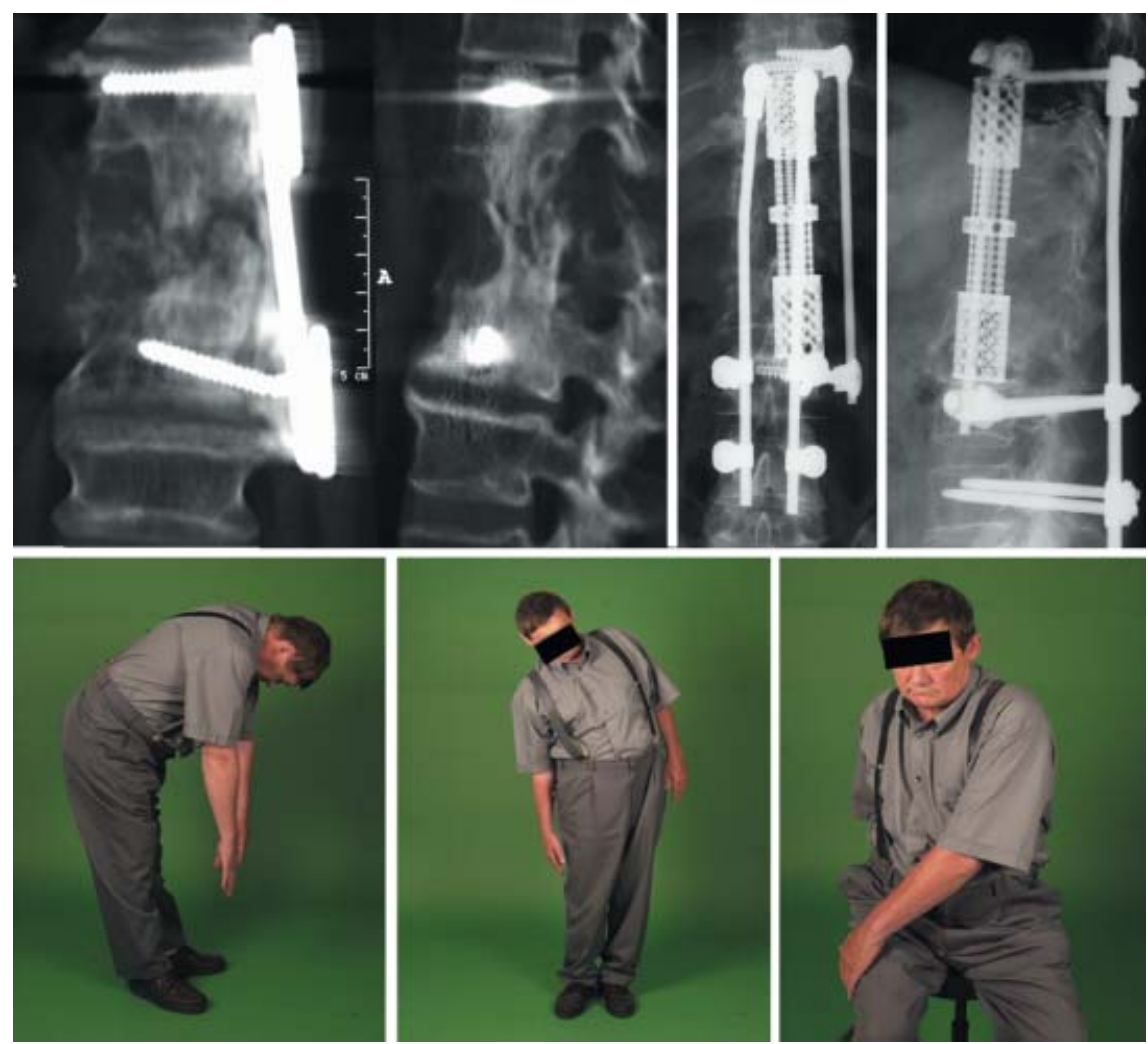

Abb.3 Rezidivbefall durch Echinokokkus nach Ablauf von fünf Jahren mit Paraparese, Spanfraktur und Implantatversagen. Primär Laminektomie und dorsale Stabilisierung, sekundär ventrale Revision und Rekonstruktion durch Wirbelkörperersatz plus Instrumentation. 

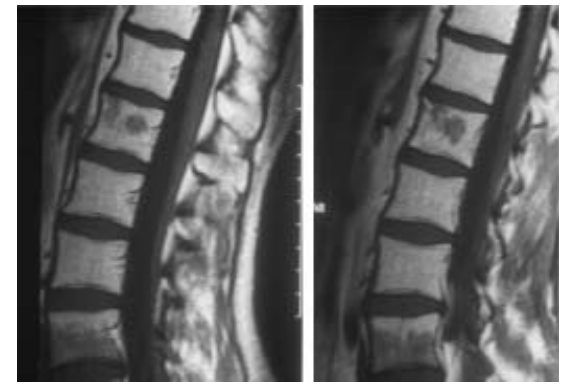

Abb. 4a Zustand nach Strahlen-/Chemotherapie eines Ewing-Sarkoms der Wirbelsäule mit Signalalteration der spongiösen Strukturen. Im Verlauf aufgetretene größenprogrediente Raumforderungen im vorbestrahlten Bereich.

Ein kombiniertes ventrodorsales Vorgehen ist sinnvoll bei Patienten mit tumorösem Befall ventraler und dorsaler Strukturen bei prognostisch als günstig einzuschätzendem Primärtumor, die kombiniert ventrodorsale Instrumentation ist hierbei erforderlich (Abb.3 u.4).
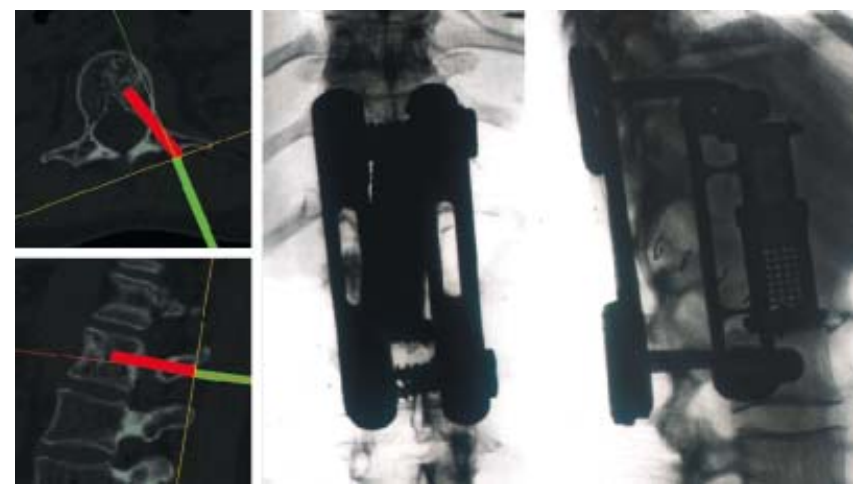

Abb. 4b Navigationsgestützte transpedikuläre $\mathrm{PE}$, kombiniert ventrodorsale Operation. Rekonstruktion der ventralen Säule über Wirbelkörperersatz. Zusätzliche Instrumentation über ein Implantatsystem mit Vierpunktabstützung.

Da Metastasen in der Wirbelsäule in der Regel im Wirbelkörper lokalisiert sind, wäre der ventrale Zugang zur Metastasenresektion prinzipiell zu bevorzugen. An Brust- und Lendenwirbelsäule mit Hauptlokalisation im thorakolumbalen Übergang bedeutet dieses in der Regel die Durchführung eines transthorakalen Zuganges. Auch unter Berücksichtigung minimalinvasiver Verfahren muss als wesentlicher Nachteil die höhere Belastung des Patienten durch pulmonale Komplikationen gesehen werden.

\section{Dr. med. Erich Hartwig Oberarzt \\ Michael Schulte \\ Oberarzt \\ Markus Schultheiss \\ Assistenzarzt \\ Michael Kramer}

Assistenzarzt

Prof. Dr. med. Lothar Kinzl

Ärztl. Direktor

Universität Ulm

Klinik für Unfall-, Hand- und Wieder-

herstellungschirurgie

Steinhövelstraße 9

D-89075 Ulm 